\title{
Small Non-coding RNAs Associated with Viral Infectious Diseases of Veterinary Importance: Potential Clinical Applications
}

\author{
Mohamed Samir ${ }^{1,2}$ and Frank Pessler ${ }^{1,3 *}$ \\ ${ }^{1}$ TWINCORE Center for Experimental and Clinical Infection Research, Hannover, Germany, ${ }^{2}$ Zoonoses Department, \\ Faculty of Veterinary Medicine, Zagazig University, Zagazig, Egypt, ${ }^{3} \mathrm{Helmholtz}$ Center for Infection Research, Braunschweig, \\ Germany
}

MicroRNAs (miRNAs) represent a class of small non-coding RNA (sncRNA) molecules that can regulate mRNAs by inducing their degradation or by blocking translation. Considering that miRNAs are ubiquitous, stable, and conserved across animal species,

OPEN ACCESS

Edited by:

Maureen T. Long,

University of Florida College of

Veterinary Medicine, USA

Reviewed by:

Jason Kindrachuk,

National Institute of Allergy and

Infectious Diseases, USA

Carol Geralyn Chitko-McKown,

United States Department of

Agriculture - Agricultural Research

Service, USA

${ }^{*}$ Correspondence:

Frank Pessler

frank.pessler@twincore.de

Specialty section:

This article was submitted to

Veterinary Infectious Diseases,

a section of the journal

Frontiers in Veterinary Science

Received: 13 December 2015

Accepted: 22 February 2016

Published: 04 April 2016

Citation:

Samir M and Pessler F (2016) Small Non-coding RNAs Associated with Viral Infectious Diseases of Veterinary

Importance: Potential Clinical

Applications.

Front. Vet. Sci. 3:22

doi: 10.3389/fvets.2016.00022 it seems feasible to exploit them for clinical applications. Unlike in human viral diseases, where some miRNA-based molecules have progressed to clinical application, in veterinary medicine, this concept is just starting to come into view. Clinically, miRNAs could represent powerful diagnostic tools to pinpoint animal viral diseases and/or prognostic tools to follow up disease progression or remission. Additionally, the possible consequences of miRNA dysregulation make them potential therapeutic targets and open the possibilities to use them as tools to generate viral disease-resistant livestock. This review presents an update of preclinical studies on using sncRNAs to combat viral diseases that affect pet and farm animals. Moreover, we discuss the possibilities and challenges of bringing these bench-based discoveries to the veterinary clinic.

Keywords: animals, animal viruses, biomarkers, clinical application, infectious diseases, miRNA, small noncoding RNAs, veterinary science

\section{INTRODUCTION}

Small non-coding RNAs (sncRNAs) are classes of short RNAs, which do not encode proteins, but rather perform regulatory functions by engaging target transcripts through sequence-specific interactions. Among these, microRNAs (miRNAs) are single-stranded molecules roughly $22 \mathrm{nt}$ in length (1). The regulatory network and function of miRNAs are based on the fact that more than one

Abbreviations: APPs, acute phase proteins; AUC, area under the curve; BFEC, bovine fetal epithelium cells; CSFV, classical swine fever virus; DMVD, degenerative mitral valve disease; FDA, Food and Drug Administration; FFPE, formalin-fixed, paraffin-embedded tissue; FMD, foot-and-mouth disease; FMDV, foot-and-mouth disease virus; GFP, green fluorescent protein; HA, hemagglutinin; HDLP, high-density lipoprotein; IAV, influenza A virus; LNA, locked nucleic acids; miRNA, microRNAs; PA, polymerase acidic; PB-1, polymerase basic 1; PPMOs, NP, nucleoprotein peptide-conjugated morpholino oligomers; PrPc , prion protein of cell; RISC, RNA-induced silencing complex; RNAi, RNA interference; ROC, receiver operating characteristic; RV, rabies virus; SA, sialic acid; shRNA, short hairpin RNA; siRNA, small interfering RNA; sncRNAs, small non-coding RNAs; SPF, specific pathogen-free; UTR, untranslated region; VEEV, Venezuelan equine encephalitis virus; WSSV, white spot syndrome virus. 
miRNA species can target the same mRNA (cooperativity) and that one miRNA can target hundreds of mRNA species (multiplicity) (2). The binding of miRNAs to the 3 ' untranslated region (UTR) of particular mRNA leads to either mRNA degradation or protein translation repression (3). miRNAs can be highly regulated both in pattern and degree of expression across multiple animal diseases. Targeting hundreds of host and pathogen encoded genes, a single miRNA can influence the gene networks essential for development and progression of a disease condition (4). This, coupled with their high degree of conservation, has made miRNAs attractive candidates for clinical application to combat pathogenic animal viruses. Being highly stable, they can be used as disease biomarkers (5). The availability of chemically synthesized miRNA mimics and agonists and vector-based RNA interference (RNAi) technology raised the idea of therapies based on non-coding RNA and made it feasible to utilize this approach to create genetically modified animal breeds that are resistant to certain viral pathogens. In this review, we summarize the current state of laboratory studies geared toward clinical applications of sncRNAs [miRNAs, small interfering RNAs (siRNAs), and short hairpin RNAs (shRNAs)] to diagnose and combat viral diseases that affect animals of veterinary importance and may thus impact animal and human health.

\section{miRNAs AS CANDIDATES FOR CLINICAL APPLICATION TO COMBAT ANIMAL VIRAL DISEASES}

\section{Potential Biomarkers}

The emerging correlation between miRNA expression and disease pathogenesis and outcomes suggests the potential use of miRNAs as biomarkers. In the first report that described the role of a miRNA as a diagnostic and prognostic marker in humans, Takamizawa et al. demonstrated that in patients with lung cancer, lower let-7 levels predicted a significantly worse prognosis after potentially curative resection (6). The intense use of advanced genomic technologies has resulted in rapid progress in human personalized medicine, where biomarker studies play a central role. Similar research interest has been emerging in veterinary medicine, albeit with some delay. Indeed, Henry et al. reported in 2010 that biomarker studies in veterinary medicine were still lagging behind those in humans (7). Biomarker research in the field of veterinary medicine focuses on the health and welfare of farm and companion animals as well as broader aspects, such as the biosafety of animal-derived food and milk production. Generally, the potential applications for biomarkers in veterinary clinics include diagnosis, staging, prognosis, and monitoring responses to therapy. Although several well-established biomarkers have been recognized for a number of veterinary viral diseases, there are still many barriers. As one example of many, lack of specificity has been recorded when using acute phase proteins (APPs) as biomarkers in pig, horse, and cattle suffering from inflammatory conditions that may have infectious etiologies, such as foot-andmouth disease virus (FMDV) infection, porcine reproductive and respiratory syndrome virus infection, pneumonia, arthritis, enteritis, and post-castration inflammation $(8-10)$. The concentration of some available biomarker molecules is affected by animal age (11). Thus, there is a need for additional, improved biomarkers for animal diseases. There is growing recognition that miRNAs may provide a specific signature that reflects the existence of a given clinical state. In this regard, the results of profiling the fluctuation of miRNA expression levels in infected organs, tissues, or single cells compared to uninfected ones throughout the course of a disease might reflect severity and outcome of the disease, including the likelihood of response to a given therapy. As biomarkers, miRNAs represent ideal candidates owing to their biological and clinical relevance, practicality, and consistent correlation with disease activity. The biological rational behind using miRNAs as biomarkers arises from their involvement in diverse physiological and pathological processes. miRNAs are extremely practical, with many advantages over other currently used biomarkers. For instance, there are efforts to develop them into diagnostics for the differentiation between viral and bacterial infections, each of which typically requires different interventions, such as quarantining versus feeding of antibiotics. The smaller number of identified miRNAs (12), compared to the approximately 30,000 protein-encoding genes currently known, implies that computational approaches dealing with miRNAs would be simpler and would require fewer resources than proteomics- or mRNA-based approaches. Another merit of miRNAs is their resistance to degradation by ribonucleases. For instance, they are stable in formalin-fixed, paraffin-embedded tissue (FFPE) independent of formalin fixation time and duration of tissue block storage (13). In contrast, mRNAs are highly fragmented and unstable in FFPE, which is problematic when FFPE is the only available sample type or when long storage of FFPE blocks has led to mRNA degradation (13). miRNAs can be detected in a large number of easily accessible samples, such as tissue biopsies, whole blood, blood cells, cerebrospinal fluid, saliva, urine, and other body fluids. Circulating miRNAs have proven to be highly resistant against RNAse activity, extreme $\mathrm{pH}$, and temperature, and certainly more so than mRNAs. This is, at least in part, because they are often contained in lipid vesicles (microvesicles and exosomes) or bound by RNA-binding proteins (5). Additionally, miRNAs resist prolonged exposure to room temperature and repeated freezing/ thawing cycles. Some miRNAs may be uniquely expressed only in specific body fluids, as exemplified by miR-224 (plasma/serum), miR-637 (tears), miR-193b (breast milk), and miR-508-5p (seminal fluid) (14). As opposed to miRNAs, proteins are a much more complex family of molecules due to use of alternate reading frames, splice variants, and various post-translational modifications, and many proteins of interest are of low abundance and/or may display major sequence variations among clinically relevant species (5).

\section{MicroRNAs as Shared Biomarkers in Human and Animal Disease}

In order to assess the role of miRNAs as a class of shared biomarkers, it is important to investigate the cross-species conservation and regulation of the same miRNA species or miRNA family. Most of the annotated miRNAs are evolutionarily conserved among a variety of organisms, particularly in their mature form, 
suggesting that the majority of miRNAs constitute a large class of predominantly orthologous or homologous molecules. As exemplified by miR-146b-5p, cross-species variation in miRNA sequence is typically observed in 1 or $2 \mathrm{nt}$ in the periphery of the mature form and in its $3^{\prime}$ UTR, i.e., away from the highly conserved seed region (Figure 1). This unique conservation pattern might be attributed to the conservation of their genomic origin. It has been reported that a consensus motif of 7-8 nt upstream and downstream of the pre-miRNA hairpin was found to be conserved among nematodes (15). Researchers from Slovenia and the USA have put together a catalog to describe the integrated assembly of intragenic miRNAs and their host genes in humans, mouse, and chicken (16). They showed that several miRNA genes were located within homologous areas, which implies that miRNA colocalization, co-expression, and potential coregulation may be conserved broadly across evolution and thus be applicable to both animal and human diseases. In the same context, previous studies reported that 300 canine miRNAs are homologs of annotated human miRNAs and that miRNA clusters are usually conserved between humans and dogs (17). Using next generation sequencing, Li et al. indicated that miRNAs in immune organs of chicken and duck were about $99 \%$ conserved (18). To gain further insights into miRNAs that are shared between species and might be used as common biomarkers, we selected a group of miRNAs that are commonly expressed upon influenza A virus (IAV) infection in humans and chicken. These miRNAs were further analyzed with miRviewer (19), a database that includes all known miRNAs of currently annotated animal genomes. William Pearson's aligning program was used to assess the degree of conservation of mature miRNAs between the two species (Table 1). The percentage of sequence identity was further confirmed by the Bioedit sequence alignment editor (20). Indeed, there is a high degree of conservation of most miRNAs between the two species (Table 1). For some miRNAs, there are sequence differences between humans and chicken in the form of deletion or addition of extra nucleotides, but these are mostly located outside the seed region. We speculate that conserved miRNAs might be the most promising candidates for universal biomarkers that may help in simultaneously pinpointing a given disease state in both species. In contrast, the non-conserved miRNAs might have the least contributory role as universal biomarkers but may play roles in more species-specific aspects of disease pathogenesis and outcomes. Apart from the sequence conservation of miRNAs, the presence of the same miRNA signatures in both humans and animals upon contracting the same infectious disease supports the concept of common biomarkers. Taken together, these observations indicate that cross-species comparisons of human and animal miRNA expression profiles as well as their conservation could provide unique opportunities to exploit miRNAs as universal biomarkers and also underline both commonalities and differences in pathology of the same disease in different species.

\section{Limitations in Using miRNAs as Biomarkers to Combat Viral Diseases}

While using miRNAs as novel biomarkers in the veterinary field represents a promising concept, it comes with unique challenges. One challenge is the presence of miRNA isomers (isomiRs), i.e., forms of miRNA that differ slightly from the annotated mature sequence. They are likely created by the enzymatic addition of adenine, cytidine, or uridine and/or imprecise cleavage by the enzymes Dicer or Drosha (21). Observational studies have shown that isomiRs can be regulated upon infection and hence are biologically and functionally meaningful (22). There is some functional overlap between miRNAs and their isomers (23). However, most miRNA annotation tools ignore these isomers by considering them as either noise or sequencing artifacts. The presence of isomiRs might affect miRNA stability and repression capability (24) and therefore reduce their value as biomarkers.

\begin{tabular}{|c|c|c|c|c|c|}
\hline Animal & & MiR-1 & $146 b-5 p$ (mature sequences) & & Accession numbers \\
\hline Human & 5 & UAGAACU & GAAUUCCAUAGGCU- - & 3 & MIMAT0002809 \\
\hline Mice & 5 & UGAGAACU & GAAUUCCAUAGGCU- - & 3 & MIMAT0003475 \\
\hline Chicken & 5 & UGAGAACU & GAAUUCCAUAGGCG- - & 3 & MIMAT0003351 \\
\hline Dog & $5^{\circ}$ & U GAGAACU & GAAUUCCAUAGGCU-- & $3^{\circ}$ & MIMAT0006667 \\
\hline Horse & 5 & UGAGAACU & GAAUUCCAUAGGCU- - & 3 & MIMAT0012891 \\
\hline Cattle & 5 & UGAGAACU & GAAUUCCAUAGGCUGU & 3 & MIMAT0009235 \\
\hline Pig & $5^{\prime}$ & UGAGAACU & GAAUUCCAUAGGC--- & 3 & MIMAT0010190 \\
\hline Goat & $5^{\prime}$ & UGAGAACU & GAAUUCCAUAGGCUGU & 3 & MIMAT0035972 \\
\hline $\begin{array}{l}\text { Atlantic } \\
\text { salmon }\end{array}$ & 5 & UGAGACU & GAAGUCCAUAGAUGG- & 3 & MIMAT0032374 \\
\hline
\end{tabular}

FIGURE 1 | Sequence alignment of the mature form of miR-146b-5p among animals and humans. The open box illustrates the high degree of conservation of the seed region of miR-146b-5p. Accession numbers are according to miRBase 21 (12). 
TABLE 1 | Sequence conservation of selected mature miRNAs in humans and chicken. ${ }^{\mathrm{a}}$

\begin{tabular}{|c|c|c|c|c|}
\hline \multirow{3}{*}{$\begin{array}{l}\text { miRNA } \\
\text { mir-29a }\end{array}$} & \multirow{3}{*}{$\begin{array}{c}\text { Identity (\%) } \\
95.2\end{array}$} & \multicolumn{2}{|c|}{ Human and chicken miRNA sequences } & \multirow{3}{*}{$\begin{array}{c}\text { Accession number } \\
\text { MIMAT0000086 } \\
\text { MIMAT0001096 }\end{array}$} \\
\hline & & Human & UAGCACCAUCUGAAAUCGGUUA & \\
\hline & & Chicken & UAGCACCAUUUGAAAUCGGUU- & \\
\hline \multirow[t]{2}{*}{ mir-18a-5p } & 100.0 & Human & UAAGGUGCAUCUAGUGCAGAUAG & MIMAT0000072 \\
\hline & & Chicken & UAAGGUGCAUCUAGUGCAGAUA- & MIMAT0001113 \\
\hline \multirow[t]{2}{*}{ mir-32-5p } & 100.0 & Human & UAUUGCACAUUACUAAGUUGCA & MIMAT0000090 \\
\hline & & Chicken & UAUUGCACAUUACUAAGUUGC- & MIMAT0001125 \\
\hline \multirow[t]{2}{*}{ mir-223-3p } & 100.0 & Human & UGUCAGUUUGUCAAAUACCCCA & MIMAT0000280 \\
\hline & & Chicken & UGUCAGUUUGUCAAAUACCCC- & MIMAT0001140 \\
\hline \multirow[t]{2}{*}{ mir-34a-5p } & 100.0 & Human & UGGCAGUGUCUUAGCUGGUUGU- & MIMAT0000255 \\
\hline & & Chicken & UGGCAGUGUCUUAGCUGGUUGUU & MIMAT0001173 \\
\hline \multirow[t]{2}{*}{ mir-142-3p } & 100.0 & Human & UGUAGUGUUUCCUACUUUAUGGA & MIMAT0000434 \\
\hline & & Chicken & UGUAGUGUUUCCUACUUUAUGG- & MIMAT0001194 \\
\hline \multirow[t]{2}{*}{$\operatorname{miR}-155-5 p$} & 100.0 & Human & UUAAUGCUAAUCGUGAUAGGGGU & MIMAT0000646 \\
\hline & & Chicken & UUAAUGCUAAUCGUGAUAGGGG- & MIMAT0001106 \\
\hline
\end{tabular}

${ }^{a}$ miRNAs are listed in ascending numerical order. Non-conserved nucleotides are printed in red. Accession numbers of miRNAs sequence are according to MiRBase 21.

When looking for miRNAs as circulating biomarkers, it is important to consider the low miRNA yield $(1-10 \mathrm{ng} / \mu \mathrm{l})$ in body fluids, such as plasma, serum, and urine (25). While some studies suggested that plasma contains a higher miRNA concentration than serum (26), a growing body of evidence has indicated that using serum as a biological sample for miRNA biomarker studies might be biased (27). This is because the stress that blood cells are exposed to during coagulation results in the release of nucleic acids, including miRNAs into the serum, which may change the true repertoire of circulating serum miRNAs giving rise to biased values. With this in mind, the lack of correlation in detection of some miRNAs in plasma and serum is not unexpected. Prior centrifugation of the blood and hemolysis might affect the amount and stability of the target miRNA and require some modifications in the isolation protocols (26). Moreover, difficulties in miRNA extraction can compromise yield and quality (28). Considering that miRNAs are differentially expressed among different animal breeds $(29,30)$, it is plausible that miRNA levels may differ among different animal breeds if they contract the same disease. This is also apparent among humans where the expression of some miRNAs was found to be related to ethnicity. In this regard, receiver operating characteristic (ROC) curve analysis indicated that let-7c predicted the onset of breast cancer with an area under the curve (AUC) of 0.99 in African Americans while having an AUC of only 0.78 in Caucasians. On the other hand, the best predictor in Caucasians was miR-589 with an AUC of 0.85 (31). This holds true for other biomarkers as well. Two reports documented a significant breed effect on the level of plasma NT-proBNP, a diagnostic marker in dogs with degenerative mitral valve disease (DMVD) $(32,33)$.

\section{RNA Interference as a Promising Tool for Therapeutic Intervention}

RNA interference is a form of post-transcriptional gene silencing that can function in a broad range of eukaryotic species. Fighting animal viruses with RNAi can be mediated by using siRNA or miRNAs, although the origin of both molecules is different. While miRNAs are endogenously produced throughout two processing steps in nucleus and cytoplasm, siRNA can be exogenously introduced directly into the cytoplasm as a double strand (34). Once in the cytoplasm, both miRNA and siRNA pass through the same processing steps where they are digested by the Dicer enzyme to form a duplex. Only one strand of this duplex is translocated into the RNA-induced silencing complex (RISC) to mediate its function (35). While siRNA forms a perfect complementarity with its target mRNA, causing its cleavage, miRNAs tend to bind to their mRNA targets less perfectly leading to repression in translation. Historically, laboratory-based experiments of using RNAi to block the replication of animal viruses started early on, namely in 2003 against IAV (36). Harnessing miRNAs for therapeutic use will rely on using gain and loss of function and is linked to the expression level of the miRNA (35). miRNAs that are beneficial for the virus and are up-regulated upon infection might be blocked using classic or modified anti-miRNAs (37). In this regard, antagomiRs (cholesterol conjugated anti-miRNAs) have been used in vitro and in vivo (38). Chemically modified nucleotides, such as locked nucleic acid (LNA), and other modifications have made it conceivable to design more stable and specific oligonucleotides. In an in vivo system, reports stated that the effect of using LNA proved to be long-lasting and safe, as neither toxicity associated with LNA nor histopathological changes were detected (39). Although there are attempts to downregulate the Dicer or Drosha enzymes as indirect ways to block miRNAs, this mechanism should be strictly controlled since blocking these enzymes will affect the entire miRNA population (40). In cases where miRNAs tend to inhibit virus replication, a therapeutic approach could be to over-express these miRNAs or to restore their levels. In this context, synthetic miRNA mimics resembling mature miRNAs that could be recognized by RISC would be a suitable tool (40). The in vivo delivery of miRNA modalities to specific cells has remained a substantial barrier. Using viruses or virus-like vectors might be innovative approaches since viruses have evolved over many generations to infect certain cells and to deliver foreign RNA, including miRNA, in a tissue- and 
cell-specific manner (41). Viral vectors can express pri-miRNA or pre-miRNA-like structures or even mature miRNA. Here, RNA viruses of both nuclear and cytoplasmic origin have been utilized (42). miRNAs may have advantages over siRNAs as therapeutic candidates. In spite of having off-target effects, miRNAs bind to their targets with partial complementarity (43) and, thus, likely tackle the high rate of mutation seen in many viruses better than siRNAs. Also, siRNAs can trigger interferon production as part of a cellular stress response pathway that can cause translation arrest, growth inhibition, and cytotoxicity (44). In contrast to the shRNA approach, the use of miRNAs enables the expression of multiple miRNAs from a single transcript as compared to only one in regular shRNA vectors. Indeed, transfection of cells with two different shRNAs may lead to competition of the two for transport and incorporation into the RISC, resulting in a reduction in shRNA processing and activity (45). Despite reports on efficient silencing of genes using RNAi, differences in the efficacy of a given vector between experiments have been reported. This might be due to inefficient cellular uptake of the RNAi and may also depend on the cell type. What follows is an overview and update of the in vitro and in vivo experiments aiming at evaluating the potential use of small RNAs, including miRNAs, as a treatment option against viral diseases that affect animals of agricultural and/or economic importance.

\section{Influenza A Virus}

Infection with IAV is a worldwide problem that affects both human and animal health $(46,47)$. The presence of multiple viral genotypes and the possibilities of antigenic shift and drift continue to raise concerns about the pandemic potential (48, 49). Current influenza vaccines and therapies have proved to be inefficient to combat the continuously evolved IAV strains due to the occurrence of antigenic variation within influenza virus genomes due to point mutations (drift) or re-assortment (shift) $(50,51)$. The emergence of resistant virus strains added another limitation to anti-IAV therapies (52). RNAi formulated in an appropriate agent would offer the potential for a new therapy by targeting viral transcripts. Furthermore, inserting a let-7b response element within the $\mathrm{H} 1 \mathrm{~N} 1$ genome created an attenuated strain that conferred protection in mice against challenge with a lethal strain, suggesting that the attenuated strain might serve as a live-attenuated vaccine (53). Around 13,500 possible siRNA target sites are present in the IAV genome. Recent reports described the usefulness of methods and procedures to select highly effective influenza-specific siRNAs in cell culture, mice, and ferrets (54). Using in silico approaches, Raza and colleagues identified five conserved amino acid sequences, three in the hemagglutinin (HA) gene (RGLFGAIAGFIE, YNAELLV, and AIAGFIE) and two in the neuraminidase (N) (RTQSEC and EECSYP) gene, which might provide potential RNAi-based therapeutic targets in various IAV strains (55). RNAi has been shown to be effective in suppressing IAV replication both in vitro and in vivo. For instance, transfecting MDCK cells with siRNA specific for nucleoprotein (NP, nucleotide positions 1496-1514) or polymerase acidic (PA, nucleotide positions 2087-2106) mRNA sequences inhibited IAV replication (36). Moreover, a mixture of siRNAs specific for highly conserved regions of NP and PA can protect mice from lethal challenge with IAV of the H5 and H7 subtypes [e.g., Ref. (56)]. siRNA against the matrix 2 (M2) gene exhibited similar or slightly higher reduction in virus replication in MDCK cells and in human HEK293 cells (57). Likewise, IAV titers in MDCK cells and in embryonated eggs were reduced more than 50 - and 100 -fold, respectively, when shRNA targeting the polymerase basic 1 (PB1) gene was transfected in vitro and in vivo using a liposome-encapsulated pSIREN/PB1 vector. In mice, the survival rate ranged between 50 and $100 \%$ (58). In another experiment, siRNA targeting a region of the M1 gene between nucleotides 331 and 351 was found to be the most effective in inhibiting M1 protein translation in cell lines. Inhibiting the viral M1 protein using this siRNA caused an $80 \%$ reduction in viral titers in supernatants of siRNAtransduced MDCK cells at 6, 8, and 10 hpi. Furthermore, virus budding ability was reduced by $40 \%$, suggesting the ability of siRNA targeting the M1 protein to suppress IAV replication (59). Another report demonstrated the efficacy of anti-NP and antiPA shRNAs in reducing IAV titers in MDCK cells and in avian $\mathrm{CH}-\mathrm{SAH}$ cells. Significant decreases of up to $80 \%$ in the levels of IAV NP mRNA and up to 370-fold in viral titer were observed in the CH-SAH cells. The approach also worked well in MDCK cells, as demonstrated by significant decreases up to $90 \%$ in the level of viral mRNA, and up to 106-fold in IAV infective titer. Furthermore, the authors identified a novel, highly efficient, and conserved RNAi target site in the viral NP gene, which can be used in antiviral cocktails of shRNAs to prevent IAV escape from RNAi silencing (60). Zhou and colleagues investigated the silencing effect of M2 and NP-specific siRNAs on IAV (H5N1, H1N1, and $\mathrm{H} 9 \mathrm{~N} 2$ ) replication in cell lines and mice (61). In the cell lines, a $0.51-1.63 \mathrm{TCID}_{50}$ reduction in virus titers was observed, and delivery of pS-M48 and pS-NP1383 significantly reduced lung virus titers in the infected mice (16- to 50-fold reduction in titer) and partially protected them from lethal IAV challenge. As an alternative approach, targeting host cell genes that are crucial for IAV replication can be conducted to control the virus. Expression of $\alpha 2,3$-linked (avian-type) and $\alpha 2,6$-linked (human-type) sialic acid (SA) receptors on host tissues is considered one of the host range and tissue tropism determinants of influenza viruses. An siRNA duplex was used to inhibit IAV binding and internalization via silencing ST6GAL1 gene that encodes the $\beta$-galactoside $\alpha$-2,6-sialyltransferase I (ST6Gal I), a protein important in SA receptor formation (62). In addition, targeting cellular proteases has been discussed as a method to suppress IAV replication. Rogers and colleagues studied pulmonary miRNA expression in mice infected with the IAV H5N1 strain and verified that furin, a member of the convertase family that mediates cleavage of hemagglutinin, is a target gene for miRNAs upon $\mathrm{H} 5 \mathrm{~N} 1$ infection (63). This highlights the importance of using miRNAs as potential therapeutic agents against IAV.

\section{Venezuelan Equine Encephalitis Virus}

Venezuelan equine encephalitis virus (VEEV) belongs to the genus alphavirus in the family Togaviridae. This virus is still endemic in many parts of the world and is considered an emerging disease threat in other parts as well as a potential biological weapon (64). So far, there are no US Food and Drug 
Administration (FDA) approved drugs or vaccines against VEEV. Thus, developing artificial miRNAs that can be used to control VEEV infection is a step in the right direction. Indeed, VEEV has been targeted efficiently by siRNA (65). Most recently, it was shown that targeting the viral non-structural protein-4 (nsp-4) region with miRNAs in BHK-21 cells efficiently inhibited viral replication, with artificial miR-3 having the greatest effect (66). This study indicated that these artificial miRNAs merit further testing in animal models for antiviral therapies against VEEV infection.

\section{Foot-and-Mouth Disease Virus}

Foot-and-mouth disease (FMD) is a highly infectious viral disease that usually affects cloven-hoofed animals. The direct impact of an FMD outbreak includes great losses to agricultural production and disruption of local economies, while the indirect effects lie in the disease control measures at both local and global levels and the high cost of disease control and prevention programs. FMDV has an RNA genome and many serotypes, and targeting conserved viral genes, such as 3D, VP4, and $2 \mathrm{~B}$, is a major aim in order to control FMD (67). The use of peptide-conjugated morpholino oligomers (PPMOs) and miRNAs with sequences complementary to various segments of the FMDV genome effectively blocked viral replication in cell culture models (68). Likewise, DNA vector-based RNAi technology can specifically suppress the expression of the VP1, $3 \mathrm{D}, \mathrm{VP} 4$, and $2 \mathrm{~B}$ genes and thus inhibit viral replication in vivo and in vitro $(67,69)$. Using adenovirus-based vectors to express siRNA molecules in cell lines and mice, Kim et al. suggested to apply RNAi treatments before and after infection with FMDV (70). Treatment after FMDV infection inhibited viral replication effectively, but a combination of treatment before and after infection gave the best results in pig kidney cells, IBRS-2 cells, and in suckling mice, as evidenced by lower viral titers in cell lines and higher survival rates of the treated mice. These experiments did reveal that the RNAi method took considerable time to induce a silencing effect, which ranged from 24 to $48 \mathrm{~h}$ (71, 72). This is considered a limitation when attempting to control certain rapidly spreading contagious diseases, including FMD, as viral spread will be faster than the inhibitory action of the RNAi. Finally, the use of artificial miRNAs (amiRs) resulted in specific silencing of reporter genes fused to FMDV target sequences (73).

\section{Classical Swine Fever Virus}

Classical swine fever virus (CSFV) can cause a hemorrhagic disease in pigs characterized by disseminated intravascular coagulation, thrombocytopenia, and immunosuppression (74, 75). CSFV has been recognized for nearly 200 years and now appears to have been eradicated in Europe and North America due to vaccinations and other control measures. The first study of using siRNA in blocking CSFV replication was conducted in 2008 (76). Three siRNA molecules targeting different regions of the CSFV Npro and NS5B genes were prepared and transfected into PK-15 cells. They caused a 4 - to 12 -fold reduction in viral genome copy number. In another study, synthetic siRNA transfected into swine kidney cells (SK-6) could target nucleotides 1130-1148 in the nucleocapsid protein $(\mathrm{C})$ of the CSFV with subsequent reduction in viral titer compared to either mock-treated or non-treated cells (77). This emphasizes the potential of siRNA to inhibit CSFV replication. Clearly, in vivo experiments need to be conducted to confirm this effect.

\section{Rabies Virus}

Rabies is a zoonotic disease caused by rabies virus (RV), a member of the Rhabidoviridae family. The disease typically infects canines (78) and is usually transmitted by animal bites, causing a lethal encephalitis. The annual number of deaths due to rabies has been estimated to be approximately 59,000 (79). The control of RV in wild carnivores has moved from culling operations to parenteral and oral vaccination of susceptible species (80), but inhibiting viral replication with siRNA or miRNAs may be another promising approach. Cell lines have been used to assess the usefulness of siRNA in inhibiting RV replication either by using a pool of siRNAs (81) or by single and multiple artificial miRNA targeting RV nucleocapsid (N) (45). In these in vitro assays, there was a comparable virus reduction at $72 \mathrm{~h}$ post-infection, especially when a single miRNA completely matched the target. Similar results were reported by others [e.g., Ref. (82)]. In cultured cells and murine model, RV glycoproteins were proved to be essential for trans-synaptic viral spread between neurons (83). This observation encouraged other researchers to target the genes encoding such glycoproteins. Sonwane et al. studied the ability of adenovirus-based siRNAs, delivered to BHK-21 cells, to inhibit RV replication and subsequently tested this approach in mice (84). In this study, siRNA inhibited viral replication in cell lines and mice. In BHK-21 cells, siRNA targeting the $\mathrm{RV}$ polymerase gene (L gene) was found to be more effective than siRNA targeting the RV NP (N gene) in inhibiting and reducing RV replication. Specifically, a $48.2 \%$ reduction of RV foci was seen in cells, in which the $\mathrm{L}$ gene was targeted versus a $41.8 \%$ reduction when the $\mathrm{N}$ gene was targeted. A significant, even greater, difference was observed at the mRNA level (17.8versus 5.7-fold reduction). In mice, inoculation of both siRNA vectors resulted in a $50 \%$ protection against a subsequent lethal RV injection. siRNAs simultaneously targeting the glycoprotein $\mathrm{G}$ and $\mathrm{N}$ genes led to an $87 \%$ reduction in viral release, demonstrating that siRNAs directed against different targets may act synergistically and increase efficacy of siRNA-based interventions against RV (85). Taken together, the above results do suggest that use of siRNAs constitutes a promising approach to interventions against RV.

\section{Viral Diseases of Fish}

Viral infection in fish aquaculture can be devastating and costly (86). Early reports of RNAi-based treatments described use of this technology in fish and shellfish in 2008 (87). In fish betanodavirus, there are two amino acid residues in the B2 protein (R53 and R60), which bind viral RNA to circumvent the RNAi pathway, underscoring the importance of the antiviral role of the host RNAi machinery (88). Dang et al. showed an inhibitory effect of siRNA on seabream iridovirus, a marine fish 
virus (89). In this study, siRNA introduced into cells infected with red seabream iridovirus specifically and effectively bound to mRNA encoding the virus major capsid protein, leading to a reduction in the production of virus particles in the supernatant of virus-infected cells, as compared to the cells receiving the control treatment. These results provide encouraging evidence that siRNA technology might be used to control fish viral diseases. More recently, a shRNA construct was found to inhibit the proliferation of viral hemorrhagic septicemia virus by targeting its $G$ gene in a sequence-specific manner (90). Infection with herpesvirus 3 causes severe financial losses in the common carp and koi culture industries worldwide (91). Although most investigations have employed in vitro approaches, RNAi might be a promising tool to combat herpesvirus 3 in carp. For instance, a pool of siRNAs specific for DNA enzyme synthesis and capsid proteins of cyprinid herpesvirus 3 virus can be a potential inhibitor of virus replication in carp fibroblasts (92). Along the same line, Gotesman et al. demonstrated that siRNAs can inhibit the thymidine kinase and DNA polymerase genes of cyprinid herpesvirus 3, causing decreased release of viral particles from transfected common carp brain cells (93). Viral infection in shrimp constitutes a great problem, and excellent reviews have discussed the use of RNAi in controlling various viral infections in shrimp [e.g., Ref. (94-96)].

\section{Potential Use of RNAi to Create Genetically Engineered Virus-Resistant Animals}

Genetic selection has been successful in mediating remarkable progress in livestock improvement. Genetic engineering of livestock is commonly used to produce pharmaceuticals or to enhance production characteristics of animals but has also proven to be important in producing animals with infectious disease resistance. For example, cows have been genetically engineered to be resistant against Staphylococcus aureus-induced mastitis (97), and laboratory investigations have been conducted with regard to creating $\alpha$-herpesvirus-resistant livestock (98). Furthermore, there are efforts to create livestock resistant against gastroenteritis coronavirus infection, but published studies are limited to work with mice (99). Against IAV infection, two potent lentivirus-based shRNAs targeting the NP and PA genes of IAV were used to generate IAV-resistant mice (100). However, a successful challenge experiment has not been reported in this system. Subsequent studies based on inhibiting genes of other pathogens have been conducted (61). With improved RNAi techniques, it is conceivable that genetically engineered diseaseresistant animals, based on siRNA or shRNA technology, may someday become reality in veterinary infectious disease medicine. Even prion diseases have been the target of transgenicanimal technology featuring shRNAs. Golding and colleagues attempted the use of siRNA technology to generate prion-resistant goat and cattle (101). First, they designed a lentivirus-based shRNA tagged with green fluorescent protein (GFP), which was directed against caprine prion protein precursor $\left(\mathrm{PrP}^{c}\right)$ mRNA and then transfected this vector into an adult goat fibroblast cell line. These cells were then used for somatic nuclear transfer to produce transgenic goat embryos for subsequent in vitro differentiation in various stages of pre-implantation development. They confirmed the silencing capacity of shRNA in brain tissue of the growing fetus compared to an age-matched normal fetus. The authors observed an approximate $90 \%$ reduction in the expression of $\mathrm{PrP}^{c}$. However, clinical efficacy in reducing the risk of a neurodegenerative disease was not determined, and data regarding efficacy were not presented. This suggests that this technique had surpassed a major technical hurdle. Furthermore, two studies described the efficacy of RNAi to silence FMDV in transgenic bovine fetal epithelium cells (BFEC), although rigorous negative controls were lacking, making it difficult to ascribe any effects to the transgenic manipulations. The first of these was conducted by Wang et al., who describe the construction of three recombinant lentiviral vectors containing shRNA against VP2 (RNAi-VP2), VP3 (RNAi-VP3), or VP4 (RNAi-VP4) of FMDV and subsequent testing of their silencing power in both 293 and BHK-21 cells (102). The lenti-RNAi-VP4 vector was transfected into bovine fetal fibroblast cells. The stably transfected cells were transferred into enucleated oocytes, and the reconstructed embryos were then transferred to recipient cows. shRNA expressed in transgenic fetuses significantly degraded viral RNA after inoculation with FMDV at a titer of 100 TCID $_{50}$ and inhibited viral replication. Thus, primary transgenic bovine fetus tongue epithelium cells became much more resistant to FMDV challenge. In the second report, a shRNA-expressing lentiviral vector targeting VP1 of FMDV resulted in strong suppression of VP1 protein expression in $293 \mathrm{~T}$ cells and also significantly inhibited viral replication in BHK-21 cells (103). The construct was then transfected into bovine fetal fibroblast cells. Cloning these somatic cells resulted in 3-month-old transgenic fetuses. FMDV RNA synthesis and viral replication were significantly reduced in primary tongue epithelial cells from the transgenic fetuses, suggesting that RNAi technology can be potentially used to generate transgenic cattle resistant against FMDV. Taken together, the studies summarized above support the idea that transgenic cloning may prove to be a useful tool to deliver antiviral and anti-prion RNAi to the germ line of animals of veterinary importance, but substantial additional work remains to be done before this technology may demonstrate efficacy in veterinary practice.

\section{REMAINING CHALLENGES}

Despite the excitement about utilizing non-coding RNAs to combat animal viral diseases, considerable challenges still need to be overcome before they can be used clinically. Animal breeders tend to rear their flocks in large groups under intensive or semi-intensive husbandry or on large farms. It would be wasteful in terms of money, time, and labor to deliver these expensive molecules on an individual basis. In this case, most veterinarians prefer to use antiviral therapies in a common source bio-vehicle, for instance, food, water, or air, to ensure quick accessibility. We think that using individual miRNA-based therapies will be more practical in special cases, such as the following: race horses, the very expensive parent flocks of chickens and turkeys that are 
intended for production of specific pathogen-free (SPF) eggs, purebred domestic animals kept as stock for distributing semen for artificial insemination, and cross breeding and improving certain animal traits for meat, milk, or fat production. Controlling contagious viral diseases, for instance, FMDV and IAV, necessitates a rapid intervention strategy to prevent virus spread from one farm to another and from animals to human. In this regard, RNAi that produces the inhibitory effect within 1 or 2 days in cell lines is considered to be insufficient, and a more rapidly operating approach is needed. Another technical challenge is that the excessive levels of the introduced miRNAs can saturate the internal host processing machine for other host small RNAs giving rise to toxicity, pathology, and mortality to the animal under therapy (104). Therefore, the dose of the introduced RNAi-based therapy should be well controlled. The delivery of the RNAi molecule is a key roadblock in this whole process. This is because RNAi molecules are negatively charged and do not penetrate the cell membrane effectively, a step that is necessary for subsequent silencing of mRNAs in the cytoplasm (105). Additionally, they may be quickly excreted, of low stability, non-tissue specific, and may have an inefficient intracellular release (106). Although the delivery of the silencing molecule may be mediated via vectors, suboptimal vector selection might reduce the silencing effect. Many delivery systems, such as nanoparticles, cationic lipids, calcium phosphate, antibodies, cholesterol, and viral vectors, have been tested (107). From another perspective, the use of a single RNAi silencing molecule with a low percent match with the target mRNA would lead to a poor target reduction. Possible solutions include either applying only one siRNA which is 100\% identical to the sequences of interest or applying more than one siRNA sequence targeting different conserved regions of the target gene. In the case of IAV, spontaneous mutations were estimated to occur at a rate of approximately $1.5 \times 10^{-5}$ per nucleotide per infection cycle (50), suggesting that target sequence mismatches will arise inevitably. Another challenge is to develop a universal RNAi molecule against the same sequence in multiple influenza strains. Some viruses may evolve mechanisms to circumvent the targeting RNAi molecule, either by expressing virus-encoded suppressors or by mutation (108). In order to avoid this, scientists have tried to design RNAi molecules that simultaneously target several sequences within a viral gene (109). In practice, in the fish aquaculture system, RNAi-based therapy have demonstrated some limitations. As a rearing system in some fish farms, the rearing cages are kept floating in the sea or river water, the so-called open sea or river cage aquaculture. Under such system, introducing RNAi molecules into fish feed will allow settlement of the uneaten food, containing the therapy, to the bottom of the water body. This would be ineffective and would also make the feed available to non-target organisms (110). Thus, an alternative improved approach would be to use RNAi in land-based ponds or tanks, owing to their direct accessibility to fish and the easy disposal of waste materials. The commercial field application of injectable therapy is neither practical nor realistic, especially with shrimp, which are reared in an intensive system. Despite its relatively high expense, soaking the shrimp in a solution containing the RNAi silencing molecule is a more practical way to ensure that an effective suppression of the gene is achieved (111). Unfortunately, there are no shrimp cell lines available for the research community, delaying a better understanding of the RNAi application in shrimp farms. Effective design of the RNAi molecule is also of special concern. Although various computational tools have been developed to systematically evaluate the targets for miRNAs and or siRNA (112-114), non-specific off-target effects need to be anticipated. The many parameters that influence specificity of miRNAs/siRNAs include the selected target region, size, the starting nucleotide, GC content, the thermodynamic properties of the introduced molecule, and the presence of internal repeats. Apart from an effective design, the use of accurate positive and negative controls is necessary to ensure the validity of RNAi data (115).

\section{FUTURE DIRECTIONS}

From the evidence gathered thus far, we have every reason to be optimistic about the future use of sncRNAs in the diagnosis, monitoring, and treatment of animal viral diseases. Zoonotic viruses continue to pose a public health threat to humans. There are miRNAs that are associated with zoonotic viral diseases that were found to be conserved among the human and animal reservoirs and exhibit similar tissue tropism. It is import to investigate both the contribution of these miRNAs to the zoonotic nature of diseases and their potential roles as biomarkers or therapeutic tools for humans and animals. This is even more important for viral diseases affecting poultry populations that are reared under both intensive and semi-intensive systems, where the pathogens can be transmitted in a short time to populate the environment and infect susceptible hosts. Regarding the use of RNAi in combating viruses, the search for a target sequence conserved across strains is of highest priority in studies targeting animal viruses, in particular, those featuring rapid genomic changes, such as IAV and other RNA viruses. However, using a pool of various siRNAs or a cocktail of siRNAs specific for virus and host genes might reduce escape of mutant viruses. In addition, it would be valuable to develop more rapidly acting RNAi technology to inhibit spread of highly contagious infections, such as FMD. Prospectively, incorporating the RNAi molecule into animal feed or the water supply might be a practical choice for the treatment of animals reared in large numbers, such as fish or poultry. Using this strategy, successful experiments have been recorded in shrimp infected with white spot syndrome virus (WSSV) (116). In spite of the extensive efforts toward formulating a suitable vehicle, one that delivers the smallest RNAi quantity in a non-toxic way remains to be discovered. In this respect, the use of a natural exosome or a natural or synthetic high-density lipoprotein (HDLP) is a novel and promising approach. These are just a few areas of research that are likely to engage veterinary scientists and virologists for years ahead. These and other improvements should further facilitate the use of miRNA and siRNA to prevent and control animal viruses at veterinary clinical sites and in the field.

\section{CONCLUSION}

Small non-coding RNAs have been known as crucial regulators of gene expression, and they have great potential for applications in the diagnosis, prevention, and treatment of 


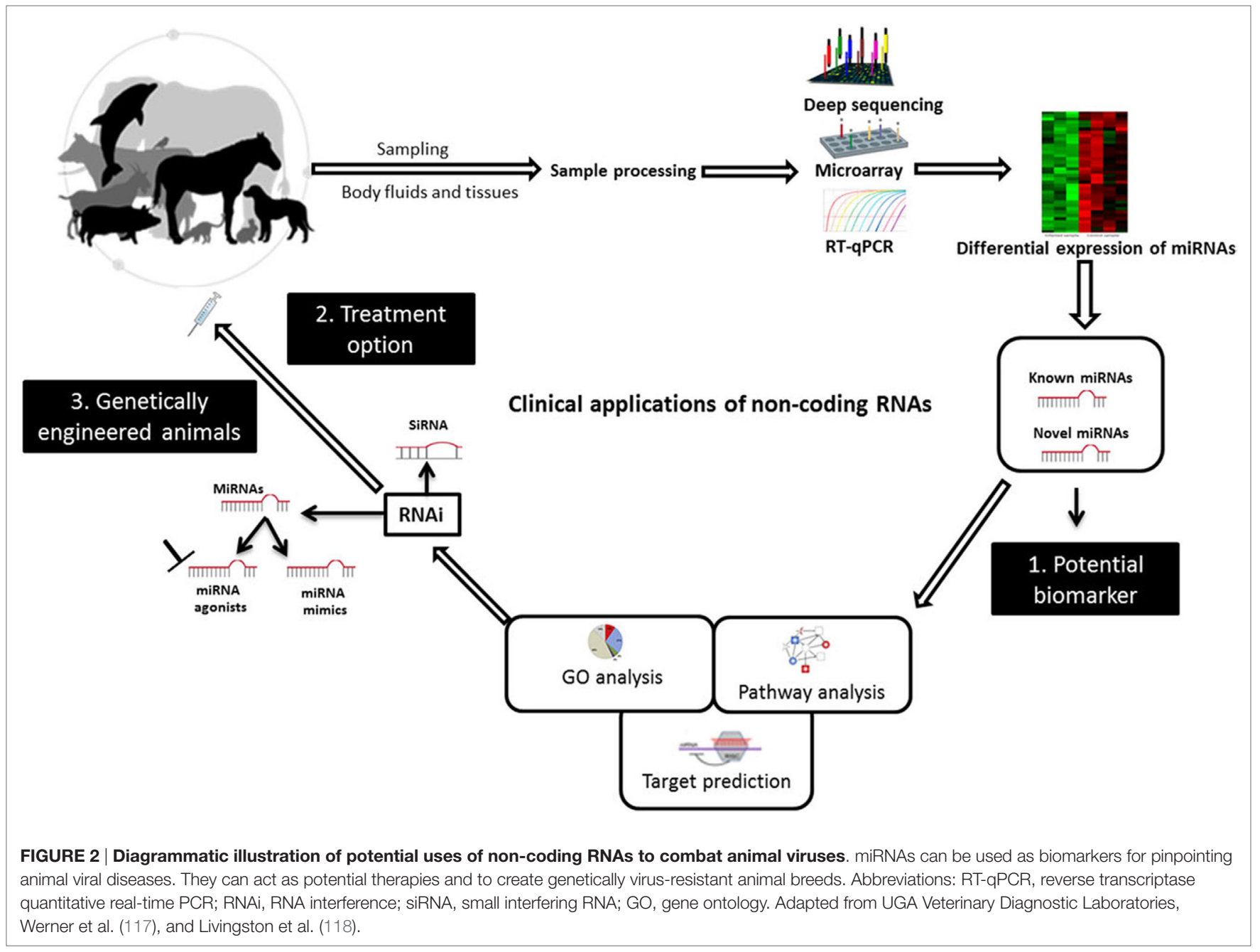

viral infectious diseases of veterinary importance. Research efforts are continuing to translate RNAi technology with its two arms, miRNAs and siRNA, to clinical applications in veterinary medicine (Figure 2). In this respect, the deregulation of miRNAs upon infection, their stability, and tissue specificity have made their study as biomarkers a fruitful area of research. siRNA molecules together with miRNA mimics or agonists can be delivered to the infected animal as a treatment option. Although there are currently no genetically engineered virus-resistant animals, the likelihood of exploiting RNAi technology, including miRNAs, is growing and is expected to help attain this aim. Bringing these molecules to the market will remain to be challenging and many barriers still need to be overcome. In fact, in vitro models would enable more detailed studies on the clinical relevance of these molecules. However, experimental animal models and infections of natural hosts in laboratory investigations will afford more realistic insights into the best ways to utilize sncRNAs to improve animal health. Importantly, developing animal-specific databases that contain experimentally validated small RNA molecules and related functional analysis will facilitate using these data for future research. The continual emergence of zoonotic viruses warrants effective collaborations between physicians and veterinarians in this issue. The available evidence suggests that the clinical use of sncRNAs in combating animal viruses may be possible in the not too distant future.

\section{AUTHOR CONTRIBUTIONS}

MS did the literature search, wrote the initial draft of the manuscript, and prepared the figures and tables. FP oversaw the project, edited the manuscript including the final version, and takes responsibility for the integrity of the data.

\section{FUNDING}

This study was supported by a German-Egyptian Research Long Term Scholarship (GERLS), a joint program between the German Academic Exchange Service (DAAD) and the Egyptian Ministry of Higher Education and Scientific Research (grant ID: A/11/92510) to MS, and by iMed, the Helmholtz Association's Initiative on Personalized Medicine (to FP). 


\section{REFERENCES}

1. Bartel DP. MicroRNAs: target recognition and regulatory functions. Cell (2009) 136(2):215-33. doi:10.1016/j.cell.2009.01.002

2. Scaria V, Hariharan M, Maiti S, Pillai B, Brahmachari SK. Host-virus interaction: a new role for microRNAs. Retrovirology (2006) 3:68. doi:10.1186/1742-4690-3-68

3. Sun G, Yan J, Noltner K, Feng J, Li H, Sarkis DA, et al. SNPs in human miRNA genes affect biogenesis and function. RNA (2009) 15(9):1640-51. doi:10.1261/rna.1560209

4. Croce CM. Causes and consequences of microRNA dysregulation in cancer. Nat Rev Genet (2009) 10(10):704-14. doi:10.1038/nrg2634

5. Etheridge A, Lee I, Hood L, Galas D, Wang K. Extracellular microRNA: a new source of biomarkers. Mutat Res (2011) 717(1-2):85-90. doi:10.1016/j. mrfmmm.2011.03.004

6. Takamizawa J, Konishi H, Yanagisawa K, Tomida S, Osada H, Endoh H, et al. Reduced expression of the let-7 microRNAs in human lung cancers in association with shortened postoperative survival. Cancer Res (2004) 64(11):3753-6. doi:10.1158/0008-5472.CAN-04-0637

7. Henry CJ. Biomarkers in veterinary cancer screening: applications, limitations and expectations. Vet J (2010) 185(1):10-4. doi:10.1016/j.tvjl.2010.04.005

8. Petersen HH, Nielsen JP, Heegaard PM. Application of acute phase protein measurements in veterinary clinical chemistry. Vet Res (2004) 35(2):163-87. doi:10.1051/vetres:2004002

9. Hofner MC, Fosbery MW, Eckersall PD, Donaldson AI. Haptoglobin response of cattle infected with foot-and-mouth disease virus. Res Vet Sci (1994) 57(1):125-8. doi:10.1016/0034-5288(94)90093-0

10. Asai T, Mori M, Okada M, Uruno K, Yazawa S, Shibata I. Elevated serum haptoglobin in pigs infected with porcine reproductive and respiratory syndrome virus. Vet Immunol Immunopathol (1999) 70(1-2):143-8. doi:10.1016/ S0165-2427(99)00069-0

11. Tóthová C, Nagy O, Kovac G. Chapter 1 - the use of acute phase proteins as biomarkers of diseases in cattle and swine. Janciauskiene S, editor. Immunology, Allergology and Rheumatology. Slovakia: InTech (2013).

12. Kozomara A, Griffiths-Jones S. miRBase: annotating high confidence microRNAs using deep sequencing data. Nucleic Acids Res (2014) 42(Database issue):D68-73. doi:10.1093/nar/gkt1181

13. Hall JS, Taylor J, Valentine HR, Irlam JJ, Eustace A, Hoskin PJ, et al. Enhanced stability of microRNA expression facilitates classification of FFPE tumour samples exhibiting near total mRNA degradation. Br JCancer (2012) 107(4):684-94. doi:10.1038/bjc.2012.294

14. Weber JA, Baxter DH, Zhang S, Huang DY, Huang KH, Lee MJ, et al. The microRNA spectrum in 12 body fluids. Clin Chem (2010) 56(11):1733-41. doi:10.1373/clinchem.2010.147405

15. Ohler U, Yekta S, Lim LP, Bartel DP, Burge CB. Patterns of flanking sequence conservation and a characteristic upstream motif for microRNA gene identification. RNA (2004) 10(9):1309-22. doi:10.1261/rna.5206304

16. Godnic I, Zorc M, Jevsinek Skok D, Calin GA, Horvat S, Dovc P, et al. Genome-wide and species-wide in silico screening for intragenic microRNAs in human, mouse and chicken. PLoS One (2013) 8(6):e65165. doi:10.1371/ journal.pone.0065165

17. Zhou D, Li S, Wen J, Gong X, Xu L, Luo Y. Genome-wide computational analyses of microRNAs and their targets from Canis familiaris. Comput Biol Chem (2008) 32(1):61-6. doi:10.1016/j.compbiolchem.2007.08.007

18. Li Z, Zhang J, Su J, Liu Y, Guo J, Zhang Y, et al. MicroRNAs in the immune organs of chickens and ducks indicate divergence of immunity against H5N1 avian influenza. FEBS Lett (2015) 589(4):419-25. doi:10.1016/j. febslet.2014.12.019

19. Kiezun A, Artzi S, Modai S, Volk N, Isakov O, Shomron N. miRviewer: a multispecies microRNA homologous viewer. BMC Res Notes (2012) 5:92. doi:10.1186/1756-0500-5-92

20. Hall T. BioEdit Version 7.0.0. Ibis Therapeutics, A Division of Isis Pharmaceuticals, Inc (2004). Available from: http://www.mbio.ncsu.edu/ bioedit/biodoc.pdf

21. Neilsen CT, Goodall GJ, Bracken CP. IsomiRs - the overlooked repertoire in the dynamic microRNAome. Trends Genet (2012) 28(11):544-9. doi:10.1016/j.tig.2012.07.005
22. Preusse M, Della Beffa C, Pessler F. Profiling of miRNA expression: RNA sequencing in the spotlight. In: Yue J, Lu L, editors. miRNAs and Human Diseases. Research Signpost (2012). p. 313-35.

23. Nicole C, Wani S, Xu Q, Gu J, Lea K, Heater S, et al. MicroRNAs and their isomiRs function cooperatively to target common biological pathways. Genome Biol (2011) 12:1-20. doi:10.1186/gb-2011-12-12-r126

24. Katoh T, Sakaguchi Y, Miyauchi K, Suzuki T, Kashiwabara S-I, Baba T, et al. Selective stabilization of mammalian microRNAs by 3' adenylation mediated by the cytoplasmic poly(A) polymerase GLD-2. Genes Dev (2009) 23(4):433-8. doi:10.1101/gad.1761509

25. Mitchell PS, Parkin RK, Kroh EM, Fritz BR, Wyman SK, PogosovaAgadjanyan EL, et al. Circulating microRNAs as stable blood-based markers for cancer detection. Proc Natl Acad Sci U S A (2008) 105(30):10513-8. doi:10.1073/pnas.0804549105

26. McDonald JS, Milosevic D, Reddi HV, Grebe SK, Algeciras-Schimnich A. Analysis of circulating microRNA: preanalytical and analytical challenges. Clin Chem (2011) 57(6):833-40. doi:10.1373/clinchem.2010.157198

27. Wang K, Yuan Y, Cho JH, McClarty S, Baxter D, Galas DJ. Comparing the MicroRNA spectrum between serum and plasma. PLoS One (2012) 7(7):e41561. doi:10.1371/journal.pone.0041561

28. Pritchard CC, Cheng HH, Tewari M. MicroRNA profiling: approaches and considerations. Nat Rev Genet (2012) 13(5):358-69. doi:10.1038/nrg3198

29. Timoneda O, Balcells I, Nunez JI, Egea R, Vera G, Castello A, et al. miRNA expression profile analysis in kidney of different porcine breeds. PLoS One (2013) 8(1):e55402. doi:10.1371/journal.pone.0055402

30. Hou X, Yang Y, Zhu S, Hua C, Zhou R, Mu Y, et al. Comparison of skeletal muscle miRNA and mRNA profiles among three pig breeds. Mol Genet Genomics (2015) 290:1-15. doi:10.1007/s00438-015-1126-3

31. Zhao H, Shen J, Medico L, Wang D, Ambrosone CB, Liu S. A pilot study of circulating miRNAs as potential biomarkers of early stage breast cancer. PLoS One (2010) 5(10):e13735. doi:10.1371/journal.pone.0013735

32. Oyama MA, Fox PR, Rush JE, Rozanski EA, Lesser M. Clinical utility of serum N-terminal pro-B-type natriuretic peptide concentration for identifying cardiac disease in dogs and assessing disease severity. J Am Vet Med Assoc (2008) 232(10):1496-503. doi:10.2460/javma.232.10.1496

33. Madsen MB, Olsen, LH, Haggstrom J, Hoglund K, Ljungvall I, Falk T, et al., Identification of 2 loci associated with development of myxomatous mitral valve disease in Cavalier King Charles Spaniels. J Hered (2011) 102(Suppl 1) S62-7.

34. Hannon GJ. RNA interference. Nature (2002) 418(6894):244-51. doi:10.1038/418244a

35. Stenvang J, Petri A, Lindow M, Obad S, Kauppinen S. Inhibition of microRNA function by antimiR oligonucleotides. Silence (2012) 3(1):1. doi:10.1186/1758-907X-3-1

36. Ge Q, McManus MT, Nguyen T, Shen CH, Sharp PA, Eisen HN, et al. RNA interference of influenza virus production by directly targeting mRNA for degradation and indirectly inhibiting all viral RNA transcription. Proc Natl Acad Sci U S A (2003) 100(5):2718-23. doi:10.1073/pnas.0437841100

37. Rossbach M. Small non-coding RNAs as novel therapeutics. Curr Mol Med (2010) 10(4):361-8. doi:10.2174/156652410791317048

38. Meister G, Landthaler M, Dorsett Y, Tuschl T. Sequence-specific inhibition of microRNA- and siRNA-induced RNA silencing. RNA (2004) 10(3):544-50. doi:10.1261/rna.5235104

39. Elmen J, Lindow M, Schutz S, Lawrence M, Petri A, Obad S, et al. LNAmediated microRNA silencing in non-human primates. Nature (2008) 452(7189):896-9. doi:10.1038/nature06783

40. van Rooij E, Marshall WS, Olson EN. Toward microRNA-based therapeutics for heart disease: the sense in antisense. Circ Res (2008) 103(9):919-28. doi:10.1161/CIRCRESAHA.108.183426

41. Pfeffer S, Zavolan M, Grasser FA, Chien M, Russo JJ, Ju J, et al. Identification of virus-encoded microRNAs. Science (2004) 304(5671):734-6. doi:10.1126/ science. 1096781

42. Tenoever BR. RNA viruses and the host miRNA machinery. Nat Rev Microbiol (2013) 11(3):169-80. doi:10.1038/nrmicro2971

43. Lam JKW, Chow MYT, Zhang Y, Leung SW. siRNA versus miRNA as therapeutics for gene silencing. Mol Ther Nucleic Acids (2015) 4:e252. doi:10.1038/ mtna.2015.23 
44. Gantier MP, Williams BR. The response of mammalian cells to double-stranded RNA. Cytokine Growth Factor Rev (2007) 18(5-6):363-71. doi:10.1016/j.cytogfr.2007.06.016

45. Israsena $\mathrm{N}$, Supavonwong $\mathrm{P}$, Ratanasetyuth $\mathrm{N}$, Khawplod $\mathrm{P}$, Hemachudha T. Inhibition of rabies virus replication by multiple artificial microRNAs. Antiviral Res (2009) 84(1):76-83. doi:10.1016/j.antiviral.2009.07.012

46. Lapinsky SE. Epidemic viral pneumonia. Curr Opin Infect Dis (2010) 23(2):139-44. doi:10.1097/QCO.0b013e328336eaae

47. Capua I, Marangon S. Control of avian influenza in poultry. Emerg Infect Dis (2006) 12(9):1319-24. doi:10.3201/eid1209.060430

48. Shimizu K. Mechanisms of antigenic variation in influenza virus. Nihon Rinsho (2000) 58(11):2199-205.

49. Guan Y, Vijaykrishna D, Bahl J, Zhu H, Wang J, Smith GJ. The emergence of pandemic influenza viruses. Protein Cell (2010) 1(1):9-13. doi:10.1007/ s13238-010-0008-z

50. Parvin JD, Moscona A, Pan WT, Leider JM, Palese P. Measurement of the mutation rates of animal viruses: influenza A virus and poliovirus type 1. J Virol (1986) 59(2):377-83.

51. Pica N, Palese P. Toward a universal influenza virus vaccine: prospects and challenges. Annu Rev Med (2013) 64:189-202. doi:10.1146/ annurev-med-120611-145115

52. Pizzorno A, Abed Y, Boivin G. Influenza drug resistance. Semin Respir Crit Care Med (2011) 32(4):409-22. doi:10.1055/s-0031-1283281

53. Feng C, Tan M, Sun W, Shi Y, Xing Z. Attenuation of the influenza virus by microRNA response element in vivo and protective efficacy against 2009 pandemic H1N1 virus in mice. Int J Infect Dis (2015) 38:146-52. doi:10.1016/j. ijid.2015.07.002

54. Seth S, Templin MV, Severson G, Baturevych O. A potential therapeutic for pandemic influenza using RNA interference. Methods Mol Biol (2010) 623:397-422. doi:10.1007/978-1-60761-588-0_26

55. Raza A, Shareef H, Salim H, Khushal R, Bokhari H. Selection of predicted siRNA as potential antiviral therapeutic agent against influenza virus. Bioinformation (2011) 6(9):340-3. doi:10.6026/97320630006340

56. Tompkins SM, Lo CY, Tumpey TM, Epstein SL. Protection against lethal influenza virus challenge by RNA interference in vivo. Proc Natl Acad Sci U S A (2004) 101(23):8682-6. doi:10.1073/pnas.0402630101

57. Sui B, Bamba D, Weng K, Ung H, Chang S, Van Dyke J, et al. The use of random homozygous gene perturbation to identify novel host-oriented targets for influenza. Virology (2009) 387(2):473-81. doi:10.1016/j.virol.2009.02.046

58. Li W, Yang X, Jiang Y, Wang B, Yang Y, Jiang Z, et al. Inhibition of influenza A virus replication by RNA interference targeted against the PB1 subunit of the RNA polymerase gene. Arch Virol (2011) 156(11):1979-87. doi:10.1007/ s00705-011-1087-8

59. Hui EK, Yap EM, An DS, Chen IS, Nayak DP. Inhibition of influenza virus matrix (M1) protein expression and virus replication by U6 promoter-driven and lentivirus-mediated delivery of siRNA. JGen Virol (2004) 85(Pt 7):1877-84. doi:10.1099/vir.0.79906-0

60. Abrahamyan A, Nagy É, Golovan SP. Human H1 promoter expressed short hairpin RNAs (shRNAs) suppress avian influenza virus replication in chicken CH-SAH and canine MDCK cells. Antiviral Res (2009) 84(2):159-67. doi:10.1016/j.antiviral.2009.08.009

61. Zhou H, Jin M, Yu Z, Xu X, Peng Y, Wu H, et al. Effective small interfering RNAs targeting matrix and nucleocapsid protein gene inhibit influenza A virus replication in cells and mice. Antiviral Res (2007) 76(2):186-93. doi:10.1016/j.antiviral.2007.07.002

62. Wu D, Huang W, Wang Y, Guan W, Li R, Yang Z, et al. Gene silencing of beta-galactosamide alpha-2,6-sialyltransferase 1 inhibits human influenza virus infection of airway epithelial cells. BMC Microbiol (2014) 14(1):78. doi:10.1186/1471-2180-14-78

63. Rogers JV, Price JA, Wendling MQ, Long JP, Bresler HS. Preliminary microRNA analysis in lung tissue to identify potential therapeutic targets against H5N1 infection. Viral Immunol (2012) 25(1):3-11. doi:10.1089/ vim.2011.0047

64. Weaver SC, Ferro C, Barrera R, Boshell J, Navarro JC. Venezuelan equine encephalitis. Annu Rev Entomol (2004) 49:141-74. doi:10.1146/annurev. ento.49.061802.123422

65. O'Brien L. Inhibition of multiple strains of Venezuelan equine encephalitis virus by a pool of four short interfering RNAs. Antiviral Res (2007) 75(1):20-9. doi:10.1016/j.antiviral.2006.11.007
66. Bhomia M, Sharma A, Gayen M, Gupta P, Maheshwari RK. Artificial microRNAs can effectively inhibit replication of Venezuelan equine encephalitis virus. Antiviral Res (2013) 100(2):429-34. doi:10.1016/j. antiviral.2013.08.010

67. Cong W, Jin H, Jiang C, Yan W, Liu M, Chen J, et al. Attenuated Salmonella choleraesuis-mediated RNAi targeted to conserved regions against footand-mouth disease virus in guinea pigs and swine. Vet Res (2010) 41(3):30. doi:10.1051/vetres/2010002

68. Du J, Gao S, Luo J, Zhang G, Cong G, Shao J, et al. Effective inhibition of foot-and-mouth disease virus (FMDV) replication in vitro by vector-delivered microRNAs targeting the 3D gene. Virol J (2011) 8:292. doi:10.1186/1743-422X-8-292

69. Chen W, Yan W, Du Q, Fei L, Liu M, Ni Z, et al. RNA interference targeting VP1 inhibits foot-and-mouth disease virus replication in BHK-21 cells and suckling mice. J Virol (2004) 78(13):6900-7. doi:10.1128/ JVI.78.13.6900-6907.2004

70. Kim S-M, Lee K-N, Park J-Y, Ko Y-J, Joo Y-S, Kim H-S, et al. Therapeutic application of RNA interference against foot-and-mouth disease virus in vitro and in vivo. Antiviral Res (2008) 80(2):178-84. doi:10.1016/j. antiviral.2008.06.001

71. Mohapatra JK, Sanyal A, Hemadri D, Tosh C, Kumar RM, Bandyopadhyay SK. Evaluation of in vitro inhibitory potential of small interfering RNAs directed against various regions of foot-and-mouth disease virus genome. Biochem Biophys Res Commun (2005) 329(3):1133-8. doi:10.1016/j. bbrc.2005.02.080

72. Liu M, Chen W, Ni Z, Yan W, Fei L, Jiao Y, et al. Cross-inhibition to heterologous foot-and-mouth disease virus infection induced by RNA interference targeting the conserved regions of viral genome. Virology (2005) 336(1):51-9. doi:10.1016/j.virol.2005.01.051

73. Gismondi MI, Ortiz XP, Currá AP, Asurmendi S, Taboga O. Artificial microRNAs as antiviral strategy to FMDV: structural implications of target selection. J Virol Methods (2014) 199:1-10. doi:10.1016/j.jviromet.2013.12.016

74. Knoetig SM, Summerfield A, Spagnuolo-Weaver M, McCullough KC. Immunopathogenesis of classical swine fever: role of monocytic cells. Immunology (1999) 97(2):359-66. doi:10.1046/j.1365-2567.1999.00775.x

75. Gomez-Villamandos JC, Carrasco L, Bautista MJ, Sierra MA, Quezada M, Hervas J, et al. African swine fever and classical swine fever: a review of the pathogenesis. Dtsch Tierarztl Wochenschr (2003) 110(4):165-9.

76. Xu X, Guo H, Xiao C, Zha Y, Shi Z, Xia X, et al. In vitro inhibition of classical swine fever virus replication by siRNAs targeting Npro and NS5B genes. Antiviral Res (2008) 78(3):188-93. doi:10.1016/j.antiviral.2007.12.012

77. Porntrakulpipat S, Supankong S, Chatchawanchonteera A, Pakdee P. RNA interference targeting nucleocapsid protein (C) inhibits classical swine fever virus replication in SK-6 cells. Vet Microbiol (2010) 142(1-2):41-4. doi:10.1016/j.vetmic.2009.09.041

78. White J, Taylor SM, Wolfram KL, O’Conner BP. Rabies in a 10-week-old puppy. Can Vet J (2007) 48(9):931-4.

79. Hampson K, Coudeville L, Lembo T, Sambo M, Kieffer A, Attlan M, et al. Estimating the global burden of endemic canine rabies. PLoS Negl Trop Dis (2015) 9(4):e0003709. doi:10.1371/journal.pntd.0003709

80. Rosatte RC. Chapter 18 - rabies control in wild carnivores. 3rd ed. In: Jackson AC, editor. Rabies. Boston: Academic Press (2013). p. 617-70.

81. Brandao PE, Castilho JG, Fahl W, Carnieli P, Oliveira RD, Macedo CI, et al. Short-interfering RNAs as antivirals against rabies. Braz J Infect Dis (2007) 11(2):224-5. doi:10.1590/S1413-86702007000200011

82. Singh NK, Meshram CD, Sonwane AA, Dahiya SS, Pawar SS, Chaturvedi $\mathrm{VK}$, et al. Protection of mice against lethal rabies virus challenge using short interfering RNAs (siRNAs) delivered through lentiviral vector. Mol Biotechnol (2013) 56(2):91-101. doi:10.1007/s12033-013-9685-1

83. Etessami R, Conzelmann KK, Fadai-Ghotbi B, Natelson B, Tsiang H, Ceccaldi PE. Spread and pathogenic characteristics of a G-deficient rabies virus recombinant: an in vitro and in vivo study. J Gen Virol (2000) 81(Pt 9):2147-53. doi:10.1099/0022-1317-81-9-2147

84. Sonwane AA, Dahiya SS, Saini M, Chaturvedi VK, Singh RP, Gupta PK. Inhibition of rabies virus multiplication by siRNA delivered through adenoviral vector in vitro in BHK-21 cells and in vivo in mice. Res Vet Sci (2012) 93(1):498-503. doi:10.1016/j.rvsc.2011.06.008

85. Meshram CD, Singh NK, Sonwane AA, Pawar SS, Mishra BP, Chaturvedi VK, et al. Evaluation of single and dual siRNAs targeting rabies virus glycoprotein 
and nucleoprotein genes for inhibition of virus multiplication in vitro. Arch Virol (2013) 158(11):2323-32. doi:10.1007/s00705-013-1738-z

86. Walker PJ, Winton JR. Emerging viral diseases of fish and shrimp. Vet Res (2010) 41(6):51. doi:10.1051/vetres/2010022

87. Schyth BD. RNAi-mediated gene silencing in fishes? J Fish Biol (2008) 72:1890-906. doi:10.1111/j.1095-8649.2008.01819.x

88. Fenner BJ, Goh W, Kwang J. Dissection of double-stranded RNA binding protein B2 from Betanodavirus. J Virol (2007) 81(11):5449-59. doi:10.1128/ JVI.00009-07

89. Dang LT, Kondo H, Hirono I, Aoki T. Inhibition of red seabream iridovirus (RSIV) replication by small interfering RNA (siRNA) in a cell culture system. Antiviral Res (2008) 77(2):142-9. doi:10.1016/j.antiviral.2007.10.007

90. Kim MS, Kim KH. Inhibition of viral hemorrhagic septicemia virus replication using a short hairpin RNA targeting the G gene. Arch Virol (2011) 156(3):457-64. doi:10.1007/s00705-010-0882-y

91. Michel B, Fournier G, Lieffrig F, Costes B, Vanderplasschen A. Cyprinid herpesvirus 3. Emerg Infect Dis (2010) 16(12):1835-43. doi:10.3201/ eid1612.100593

92. Adamek M, Rauch G, Brogden G, Steinhagen D. Small interfering RNA treatment can inhibit Cyprinid herpesvirus 3 associated cell death in vitro. Pol J Vet Sci (2014) 17(4):733-5. doi:10.2478/pjvs-2014-0108

93. Gotesman M, Soliman H, Besch R, El-Matbouli M. In vitro inhibition of Cyprinid herpesvirus-3 replication by RNAi. J Virol Methods (2014) 206:63-6. doi:10.1016/j.jviromet.2014.05.022

94. Labreuche Y, Warr GW. Insights into the antiviral functions of the RNAi machinery in penaeid shrimp. Fish Shellfish Immunol (2013) 34(4):1002-10. doi:10.1016/j.fsi.2012.06.008

95. Shekhar MS, Lu Y. Application of nucleic-acid-based therapeutics for viral infections in shrimp aquaculture. Mar Biotechnol (NY) (2009) 11(1):1-9. doi:10.1007/s10126-008-9155-0

96. Robalino J, Bartlett TC, Chapman RW, Gross PS, Browdy CL, Warr GW. Double-stranded RNA and antiviral immunity in marine shrimp: inducible host mechanisms and evidence for the evolution of viral counter-responses. Dev Comp Immunol (2007) 31(6):539-47. doi:10.1016/j.dci.2006.08.011

97. Wall RJ, Powell AM, Paape MJ, Kerr DE, Bannerman DD, Pursel VG, et al. Genetically enhanced cows resist intramammary Staphylococcus aureus infection. Nat Biotechnol (2005) 23(4):445-51. doi:10.1038/nbt0705-897a

98. Ono E, Amagai K, Taharaguchi S, Tomioka Y, Yoshino S, Watanabe Y, et al. Transgenic mice expressing a soluble form of porcine nectin-1/herpesvirus entry mediator $\mathrm{C}$ as a model for pseudorabies-resistant livestock. Proc Natl Acad Sci U S A (2004) 101(46):16150-5. doi:10.1073/pnas. 0405816101

99. Sola I, Castilla J, Pintado B, Sánchez-Morgado JM, Whitelaw CBA, Clark AJ, et al. Transgenic mice secreting Coronavirus neutralizing antibodies into the milk. J Virol (1998) 72(5):3762-72.

100. Pinard M-H, Gay C, Pastoret P-P, Dodet B, editors. Animal Genomics for Animal Health. Basel: Karger (2008).

101. Golding MC, Long CR, Carmell MA, Hannon GJ, Westhusin ME. Suppression of prion protein in livestock by RNA interference. Proc Natl Acad Sci U S A (2006) 103(14):5285-90. doi:10.1073/pnas.0600813103

102. Wang H, Wu J, Liu X, He H, Ding F, Yang H, et al. Identification of short hairpin RNA targeting foot-and-mouth disease virus with transgenic bovine fetal epithelium cells. PLoS One (2012) 7(8):e42356. doi:10.1371/journal. pone. 0042356

103. Wang H, Liu X, Wu J, Wu G, Yu L, He C, et al. Bovine fetal epithelium cells expressing shRNA targeting viral VP1 gene resisted against foot-and-mouth disease virus. Virology (2013) 439(2):115-21. doi:10.1016/j. virol.2013.02.003

104. Snove O Jr, Rossi JJ. Toxicity in mice expressing short hairpin RNAs gives new insight into RNAi. Genome Biol (2006) 7(8):231. doi:10.1186/ gb-2006-7-8-231

105. Li CX, Parker A, Menocal E, Xiang S, Borodyansky L, Fruehauf JH. Delivery of RNA interference. Cell Cycle (2006) 5(18):2103-9. doi:10.4161/cc.5.18.3192

106. Shim MS, Kwon YJ. Efficient and targeted delivery of siRNA in vivo. FEBS J (2010) 277(23):4814-27. doi:10.1111/j.1742-4658.2010.07904.x

107. Akhtar S, Benter I. Toxicogenomics of non-viral drug delivery systems for RNAi: potential impact on siRNA-mediated gene silencing activity and specificity. Adv Drug Deliv Rev (2007) 59(2-3):164-82. doi:10.1016/j. addr.2007.03.010

108. Berkhout B, Das AT. HIV-1 escape from RNAi antivirals: yet another Houdini action? Mol Ther Nucleic Acids (2012) 1:e26. doi:10.1038/mtna.2012.22

109. Zheng ZM, Tang S, Tao M. Development of resistance to RNAi in mammalian cells. Ann N Y Acad Sci (2005) 1058:105-18. doi:10.1196/annals.1359.019

110. Young ND, Crosbie PB, Adams MB, Nowak BF, Morrison RN. Neoparamoeba perurans n. sp., an agent of amoebic gill disease of Atlantic salmon (Salmo salar). Int J Parasitol (2007) 37(13):1469-81. doi:10.1016/j. ijpara.2007.04.018

111. Lima PC, Harris JO, Cook M. Exploring RNAi as a therapeutic strategy for controlling disease in aquaculture. Fish Shellfish Immunol (2013) 34(3):72943. doi:10.1016/j.fsi.2012.11.037

112. Naito Y, Yamada T, Ui-Tei K, Morishita S, Saigo K. siDirect: highly effective, target-specific siRNA design software for mammalian RNA interference. Nucleic Acids Res (2004) 32(Web server issue):W124-9. doi:10.1093/nar/gkh442

113. Qiu S, Adema CM, Lane T. A computational study of off-target effects of RNA interference. Nucleic Acids Res (2005) 33(6):1834-47. doi:10.1093/nar/ gki324

114. Xu P, Zhang Y, Kang L, Roossinck MJ, Mysore KS. Computational estimation and experimental verification of off-target silencing during posttranscriptional gene silencing in plants. Plant Physiol (2006) 142(2):429-40. doi:10.1104/pp.106.083295

115. Echeverri CJ, Beachy PA, Baum B, Boutros M, Buchholz F, Chanda SK, et al. Minimizing the risk of reporting false positives in large-scale RNAi screens. Nat Methods (2006) 3(10):777-9. doi:10.1038/nmeth1006-777

116. Sarathi M, Simon MC, Venkatesan C, Hameed AS. Oral administration of bacterially expressed VP28dsRNA to protect Penaeus monodon from white spot syndrome virus. Mar Biotechnol (NY) (2008) 10(3):242-9. doi:10.1007/ s10126-007-9057-6

117. Werner T. Bioinformatics applications for pathway analysis of microarray data. Curr Opin Biotechnol (2008) 19:50-4.

118. Livingston AD, Campbell CJ, Wagner EK, Ghazal P. Biochip sensors for the rapid and sensitive detection of viral disease. Genome Biol (2005) 6:1-5.

Conflict of Interest Statement: The authors declare that the research was conducted in the absence of any commercial or financial relationships that could be construed as a potential conflict of interest.

Copyright $\odot 2016$ Samir and Pessler. This is an open-access article distributed under the terms of the Creative Commons Attribution License (CC BY). The use, distribution or reproduction in other forums is permitted, provided the original author(s) or licensor are credited and that the original publication in this journal is cited, in accordance with accepted academic practice. No use, distribution or reproduction is permitted which does not comply with these terms. 